\title{
Le Dr. Arnold-C. Klebs
}

Le 6 mars 1943 décédait aux Terrasses, près de Nyon, le Dr. ArnoldC. Klebs, à l'âge de 73 ans.

C'était une personnalité puissante, un homme exceptionnel tant par la vivacité de son intelligence, l'intensité de ses sentiments que par les contrastes violents de son caractère, rançon d'un esprit éminent. Une longue amitié dans les bons et les mauvais jours, la sincérité absolue de ses confidences, nous l'ont fait connaître aussi intimément qu'il est possible de connaître quelqu'un. A l'homme courageux qui a voulu regarder en face l'humaine nature avec ses tares, avec ses qualités, qui s'est disséqué lui-même, pénétrant jusqu'au fond de son âme tourmentée, éclairant lucidement, avec intrépidité, les recoins d'ombre comme les côtés lumineux, on doit une égale franchise. Nous savons bien que la vérité d'un individu n'est que le reflet de la réalité dans son miroir personnel. C'est sa seule vérité et quand le miroir est de grande marque et constamment tenu au clair, son témoignage atteint une haute valeur. L'objectivité nous semble consister essentiellement à laisser au miroir le soin de donner son image sans retouche, à le polir, à l'améliorer sans cesse pour augmenter la portée de son message; à le diriger enfin vers tout ce qui est important pour qu'il soit le reflet de la totalité. Arnold-C. Klebs a pris un soin minutieux de son miroir, qui était d'exceptionnelle qualité. Il y a lu sans peur, sans complaisance, l'essentiel de sa personnalité. Il a permis à ses amis, avec l'absolue franchise de la vraie amitié, de regarder dans son miroir, d'y voir son image et la leur par surcroît. Et c'est là, peutêtre, le plus grand service qu'il ait rendu à ceux, très nombreux, qui sont venus, attirés par sa personnalité, ses connaissances étendues et la valeur de son jugement.

En lui dédiant l'un de ses livres, le $D^{r}$ William Welch parle de sa «divine insatisfaction». Il avait mis le doigt sur le trait le plus caractéristique de sa personnalité. Mais si Arnold-C. Klebs était insatisfait, ce n'était point de la situation qu'il occupait dans le monde scientifique; il ne cherchait pas à se créer un personnage et il méprisait les pontifes avec toute la fougue de son tempérament. Il pensait que la connaissance est une âpre recherche, exigeant un travail minutieux, beaucoup de méfiance de soi-même, une discipline sévère, une bonne dose d'humilité et ce désintéressement qui permet d'abandonner sans regret une opinion 
qu'on découvre erronée, sans vains remords non plus, pour ne pas briser l'élan vers de nouvelles quêtes.

Il voyait dans tout chercheur un frère, un ami qu'il faut aider de tout son pouvoir. Sa plus grande joie était de faciliter son travail dans toute la mesure de ses forces: il prodiguait les conseil, fournissait les livres et les références, ouvrait les portes. Il possédait un talent exceptionnel pour arranger les rencontres, pour nouer les liens. Il avait des amis dans le monde entier, spécialement parmi les médecins et les historiens. Son grand plaisir était de les réunir, de les faire valoir. Sa conversation était vivante, paradoxale, provoquante, pleine d'aperçus originaux. Il parlait doucement, sans gestes, méticuleusement, avec nuance, quand le tour de la conversation lui plaisait par sa sincérité, par le sérieux des opinions, la prudence des jugements, l'objectivité des affirmations. Puis brusquement, il tonnait, morigénait avec une crudité, une truculence d'expression, une violence de language sans pareille, destinée à écraser l'infâme. Il avait perçu une hésitation, une pointe de vanité ou de présomption, une maladresse d'expression, une trop grande soumission aux idées toutes faites. Il fallait détruire dans l'œuf ces péchés inexpiables, ces tendances fâcheuses. On avait déçu son sens de la perfection, on avait gâté son personnage. Il vous voulait toujours à l'extrême pointe de vous-même. Bienfaisante et tonique insatisfaction!, langage des forts épris de perfection.

Auprès de lui, on respirait l'air du large. C'était un citoyen du monde, un international. Sa culture s'étendait à tout ce qui est important, avec une prédilection pour les hommes marquants, ceux qui ont fait étape dans le passé comme dans le présent. Il aimait le phénomène humain dans ce qu'il a de meilleur, de plus raffiné. La guerre a été pour lui un déchirement intérieur.

Il abordait les êtres et les choses de deux manières bien différentes. Son premier mouvement était celui d'un homme de goût et de jugement: il saisissait l'essentiel d'une personnalité, il appréciait les circonstances avec perspicacité. C'était un conseiller sûr, allant rapidement à l'essentiel. Son second mouvement était de se reprendre. Il paraissait craindre la trop grande rapidité de son opinion première. Il fallait examiner la question sous toutes ses faces. Ne pouvait-on pas soutenir juste le contraire de ce qu'il venait de dire? Toute substance doit être pétrie, examinée sous des éclairages différents pour livrer son secret. Il semblait penser 
avec Hippocrate que "Chaque chose a en elle même son contraire. Ce qui parle a son silence, la raison a sa folie». (Du Régime, L. I.)

Par dessus tout, il avait horreur de la médiocrité.

Artiste, il s'émerveillait de la puissance expressive de la ligne. On peut comparer les deux démarches de son esprit à l'art très différent de deux nobles artistes. La première était semblable à l'art dépouillé de Pompon. La forme est simplifiée à l'extrême et c'est le jeu de la lumière qui suggère, sur les surfaces polies, le dessin d'une plume ou le velouté d'un pelage. L'essentiel est atteint par les moyens les plus simples, maîtrise obtenue au prix d'un patient labeur. Son jugement réfléchi se rapprochait davantage de l'art de Dürer: la forme est saisie dans sa complexité par une série de traits dont chacun est une partie même de la réalité. A celà aussi il avait réfléchi sans bien se douter qu'il se peignait ainsi lui-même.

Il s'étonnait parfois d'avoir pu conserver des amis malgré la violence de son caractère. C'est qu'il avait le culte de l'amitié, qu'il y mettait le meilleur de lui-même. Elle l'illuminait dans les bons jours, le transportait dans un domaine où toute petitesse, toute méfiance disparaissent. Il était chaleureux, compréhensif, un sourire inoubliable éclairait son visage. On le quittait heureux, léger, soulevé par son affection. C'était le grand, le cher Klebs qui s'était livré, celui qui reste dans le cœur de ceux qu'il avait élus: le travailleur consciencieux, l'érudit de goût, le critique sincère et franc, l'humaniste en un mot; par dessus tout, l'ami.

On ne possède pas de tels dons sans en payer la rançon. Nous parlions un jour du caractère difficile de son père, Edwin Klebs, et je lui signalai l'étude pénétrante de Schopenhauer sur le caractère des hommes de génie (Le monde comme volonté et comme représentation, trad. de A. Burdeau, tome 3, page 200). Il la connaissait et pensait qu'elle était perspicace. Elle éclaire si bien certains traits curieux du caractère d'Arnold-C. Klebs que je n'hésite pas à en transcrire quelques passages.

«Quand..., à l'occasion, cette connaissance d'une élévation démesurée se tournera toute entière, de toute son énergie, vers les intérêts et les misères de la volonté, il lui arrivera facilement d'en prendre une idée trop vive, de voir tout sous des couleurs trop crues, dans un jour trop intense, sous un grossissement énorme et l'individu ne pourra tomber que dans l'extrême... Toute grande œuvre théorique, de quelque nature qu'elle soit, demande pour être produite, de la part de son auteur, qu'il dirige toutes les forces de son énergie vers un seul point, 
qu'il les y fasse converger et les y concentre avec tant de force, de fermeté et de persistance, que tout le reste disparaisse à ses yeux et que son sujet remplisse pour lui toute la réalité.» (N'est-ce pas pour Klebs, la signification de la devise gravée sur la porte de sa bibliothèque: Se tu sarai solo, tu sarai tutto tuo?)

«Mais cette même grande et puissante concentration, l'un des privilèges du génie, se produit aussi parfois pour les objets de la réalité, pour les intérêts de la vie quotidienne; portés alors sous un tel foyer, ils acquièrent un grossissement si monstrueux qu'ils apparaissent, comme la puce vue au microscope solaire, avec les dimensions d'un éléphant. De là, parfois, chez les individus éminents, ces émotions violentes et diverses à propos de bagatelles; les autres ne conçoivent pas comment ils peuvent être jetés dans l'affliction, dans la joie, dans l'angoisse, la crainte et la colère, etc., par des choses qui laisseraient parfaitement calme un homme du vulgaire. Aussi le génie manque-t-il de sang-froid, car le sang-froid consiste justement à ne voir dans les choses que ce qui leur appartient, surtout par rapport à nos fins possibles ...»

Les plus beaux fruits sont portés par des arbres à qui la taille du jardinier, un terrain spécial, de multiples blessures et contraintes ont fait perdre la qualité d'arbres libres. Il faut couper les hautes branches, courber les rameaux vers le sol, modifier le port et l'allure pour obtenir ce résultat. La pluie s'infiltre dans les moignons, le cœur du bois pourrit, seuls restent les couches corticales et l'écorce, vecteurs de vie et d'abondance. L'arbre perd sa stature naturelle, une partie de sa dignité d'être librement épanoui; il se couvre par contre de fleurs et de fruits. Celà lui confère une autre dignité, celle d'un être spécialisé et conduit par là à une excellence partielle, acquise aux dépens de sa stature, de son harmonie, de sa solidité même. Un égoösme extérieur est intervenu pour obtenir des qualités utiles à l'homme.

Parfois, chez l'homme, l'égoïsme intérieur, la qualité exceptionnelle de certains dons, les possibilités qu'ouvrent certains défauts conduisent à des résultats semblables: ainsi qu'il l'a lui-même souvent et hautement confessé, Arnold-C. Klebs avait des défauts éclatants. Son égoïsme, sa volonté de dominer, qu'il abdiquait à l'égard des amis, sa violence, étaient des traits trop frappants pour passer inaperçus. Mais songe-t-on aux branches tordues, à la fragilité des troncs attaqués par la pluie, à la stature modifiée, devant un beau pommier couvert de ses fleurs délicates sous le soleil printanier clair et jeune, puis de beaux fruits savoureux 
sous la lumière plus douce de l'automne vieillissant? N'y a-t-il pas aussi quelque chose d'émouvant dans l'effort vain de l'écorce pour protéger les moignons mis à nu par la taille?

C'est ce que pense un ami, hanté par les souvenirs, en méditant dans les vergers fleuris d'un vieux pays qui lui aurait plu par sa grâce et la violence même de ses contrastes.

Sierre, avril 1943.

G. Piotet.

\section{Galilée et le problème du temps}

Des voix plus autorisées que la mienne célébreront ici la gloire de Galilée. N'étant pas historien, je me garderai de décrire l'un ou l'autre des épisodes de cette vie admirable. Je voudrais plutôt, après tant d'autres, situer quelques-unes des découvertes de Galilée dans le cadre des progrès de la mécanique et caractériser de mon mieux l'étape qu'il a fait franchir à la science à l'aurore des temps modernes. Bien que l'étude sur la chute des corps ait précédé les découvertes astronomiques, c'est par ces dernières que nous commencerons.

Pour les disciples d'Aristote, si nombreux encore à l'époque de la Renaissance, l'univers se composait de deux parties nettement distinctes et qui étaient chacune régies par des principes propres. Le monde terrestre, dit aussi sublunaire, donc en deça de la lune, était celui des choses corruptibles; tandis qu'au delà se mouvaient les sphères parfaites le long des épicycles de Ptolémée. Les mouvements des astres ne pouvaient être que circulaires ou composés de mouvements circulaires uniformes en vertu même de la perfection de la circonférence, partout identique à elle-même. L'observation des mouvements des astres avait été poussée très loin durant l'antiquité, puis chez les Arabes, et enfin à l'époque qui précède celle de Galilée. Les périodes du mouvement des planètes, la période lunaire, le phénomène de la précession des équinoxes, l'angle variable que fait l'axe du monde avec celui de l'écliptique, avaient été mesurés avec une précision devant laquelle on reste confondu. Des tables astronomiques étaient établies, qui permettaient de prévoir les retours des astres et constituaient une excellente connaissance des temps, comme diraient les astronomes. Mais, de quoi s'agis- 